(C) 2018 IEEE. Personal use of this material is permitted. Permission from IEEE must be obtained for all other uses, in any current or future media, including reprinting/republishing this material for advertising or promotional purposes, creating new collective works, for resale or redistribution to servers or lists, or reuse of any copyrighted component of this work in other works. 


\title{
Full EM Design Method for HTS MMIC Josephson Mixers
}

\author{
Ting Zhang, Xiang Gao, Jia Du, Yingiie Jay Guo, Fellow, IEEE
}

\begin{abstract}
We report the full electromagnetic (EM) design and simulation method, and applied it to develop a $34 \mathrm{GHz}$ High temperature superconducting (HTS) microwave monolithic integrated circuit (MMIC) Josephson mixer. The mixer is modeled in EM simulation software, High Frequency Simulation Structural Simulator (HFSS), with the junction area modeled as an excitation port with frequency-dependent impedance. Impedance matching between the junction and RF/IF ports is then optimized accordingly. Module design is carried out for the optimized HTS Josephson mixer, and the cavity resonance issue is investigated and eliminated. The HTS mixer module was experimentally developed and measured to verify the simulation. The measured frequency response of the conversion gain agrees with the simulation results of combined RF and IF transmission loss.
\end{abstract}

Index Terms-High temperature superconducting (HTS), Josephson mixer, MMIC, EM design.

\section{INTRODUCTION}

$\mathbf{H}$ TS materials have ultra-low surface resistance at frequencies below $100 \mathrm{GHz}$, which has been applied to make filters and resonators with superior performance [1-3]. The lownoise and high non-linearity properties of the HTS Josephson junctions make them ideal for the key component of mixers [47]. A series of HTS MMIC Josephson mixers, ranging from 7 $\mathrm{GHz}$ to $36 \mathrm{GHz}$, have been developed at CSIRO, Australia, of which the $\mathrm{YBa}_{2} \mathrm{Cu}_{3} \mathrm{O}_{7-\mathrm{x}}(\mathrm{YBCO})$ step-edge Josephson junctions are integrated monolithically with a series of YBCO filters to achieve better performance [8-11]. Meanwhile, modelling method of such an HTS MMIC Josephson circuit has been developed $[12,13]$, in which impedance matching and optimization theory between passive devices and Josephson junctions were investigated. The model has provided some theoretical guidance for the MMIC Josephson mixer circuit design, but it still has several limitations: First, the parameters of each passive components could only be tuned individually without including the effect caused by other parts of the circuit. Second, electromagnetic effect starts to be dominant and generate strong interferences between ports at mm-wave frequencies, which cannot be simulated in the modelling method consisting of ideal, lossless and interference-free components. A full EM

Ting Zhang is with University of Technology, Sydney, NSW 2007, Australia (e-mail: Ting.Zhang@uts.edu.au).

Xiang Gao is with CSIRO Manufacturing, Lindfield, NSW 2070, Australia (email: Xiang.Gao@csiro.au). simulation method combining the passive devices and the Josephson junction section is, therefore, highly desirable for HTS MMIC Josephson mixer design in the mm-wave range.

In this work, a full EM simulation approach is presented, and a $34 \mathrm{GHz}$ HTS MMIC Josephson mixer is designed and experimentally developed for verification of our EM design theory and comparing with the simulation results. The Josephson junction in the mixer is modelled as an excitation port with a frequency-dependent impedance, which enables a comprehensive EM simulation of the whole mixer circuit. RF and IF port impedance is optimized for better matching to the junction, while isolation between different ports is also considered in the simulation. A housing is designed to accommodate the HTS MMIC mixer chip and other relevant components. Cavity resonance of the housing is investigated and eliminated by design optimization. The Josephson mixer has been fabricated, packaged and measured for comparison with the simulation results. Modeling approaches, design considerations, impedance optimization, simulated and measured frequency response of the mixer are reported.

\section{HTS MMIC MIXER DESIGN}

Fig. 1 shows the layout of the proposed HTS MMIC Josephson mixer. All the passive and active devices are integrated onto a $4.5 \times 4 \times 0.3 \mathrm{~mm}^{3} \mathrm{MgO}$ substrate with a dielectric constant of

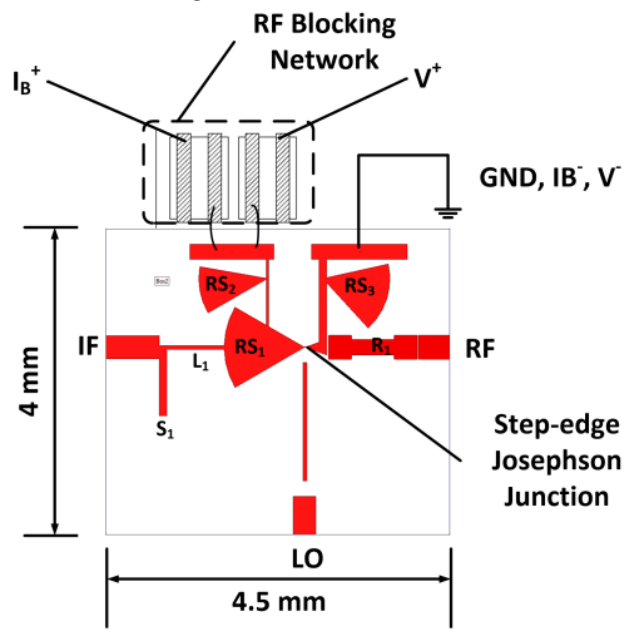

Fig. 1. Layout of the designed HTS MMIC Josephson mixer.

Jia Du is with CSIRO Manufacturing, Lindfield, NSW 2070, Australia (e-mail: Jia.Du@csiro.au).

Yingjie Jay Guo is with University of Technology, Sydney, NSW 2007, Australia (e-mail: Jay.Guo@uts.edu.au). 


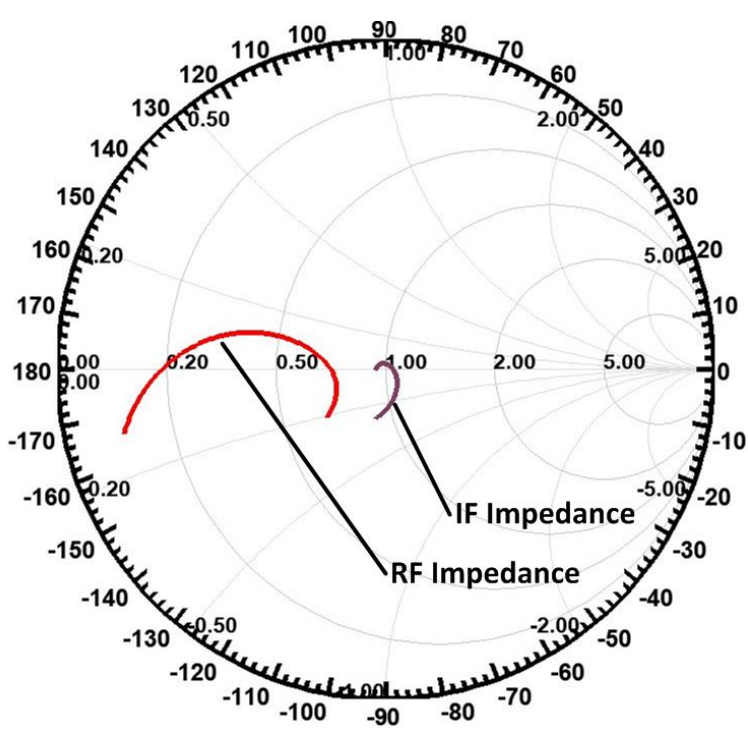

Fig. 2. Simulation result of normalized RF input impedance and IF output impedance. The junction-equivalent port impedance is $5 \Omega$ for $\mathrm{RF}$ and $50 \Omega$ for IF, respectively.

9.7. Compactness is further improved compared with our previous work at similar frequencies $[10,11]$. LO frequency is set to be $36 \mathrm{GHz}$, and the desired RF and IF frequency ranges are 33.5 to $35 \mathrm{GHz}$ and 1 to $2.5 \mathrm{GHz}$, respectively.

The design of the mixer follows the modeling theory in previous papers [6, 13], and the simulation in this work is performed using HFSS. For modeling and circuit designs at RF frequencies, the junction area is considered to be equivalent to an excitation port with $5 \Omega$ impedance, which is close to the typical step-edge junction intrinsic resistance $R_{n}$ values measured previously [11]. A quarter-wavelength radius stub $\mathrm{RS}_{1}$, is applied at the other end of the junction to provide a virtual ground for RF and LO inputs, due to the absence of physical grounding of the HTS step-edge Josephson junction's singlelayer structure. A step-impedance resonator $\mathrm{R}_{1}$ is applied at the RF input, and connected to the junction via a gap. In this way, a capacitive coupling is introduced to eliminate the impedance imaginary part introduced by the short tapered line connected to the junction for the convenience of impedance matching. The biasing network for $\mathrm{V}^{-}$and $\mathrm{I}_{\mathrm{B}}{ }^{-}$serves as an impedance matching stub for RF input, with a shunted radius stub $\mathrm{RS}_{3}$ for RF blocking. In this way, RF input impedance is tuned down to around $25 \Omega$, as shown in Fig. 2, which results in an insertion loss around $2.5 \mathrm{~dB}$. Simulation result of the insertion loss between the junction and all three ports is shown in Fig. 3. The average $\mathrm{RF}$ insertion loss is around $3 \mathrm{~dB}$ from $33.5 \mathrm{GHz}$ to $35 \mathrm{GHz}$, while the isolation from LO and IF ports are all better than 20 $\mathrm{dB}$. The designed RF layout has improved the insertion loss by over $3 \mathrm{~dB}$, compared with a direct connection between the junction and the $50 \Omega$ port using tapered line, which is approximately $7 \mathrm{~dB}$.

Impedance matching network at IF frequency will be bulky due to longer wavelengths, compared with RF frequency. Furthermore, the impedance of IF output port should better match the junction's dynamic resistance $R_{\mathrm{d}}$, which can be close to 50 $\Omega$. Therefore, the circuit design consideration at IF frequency is

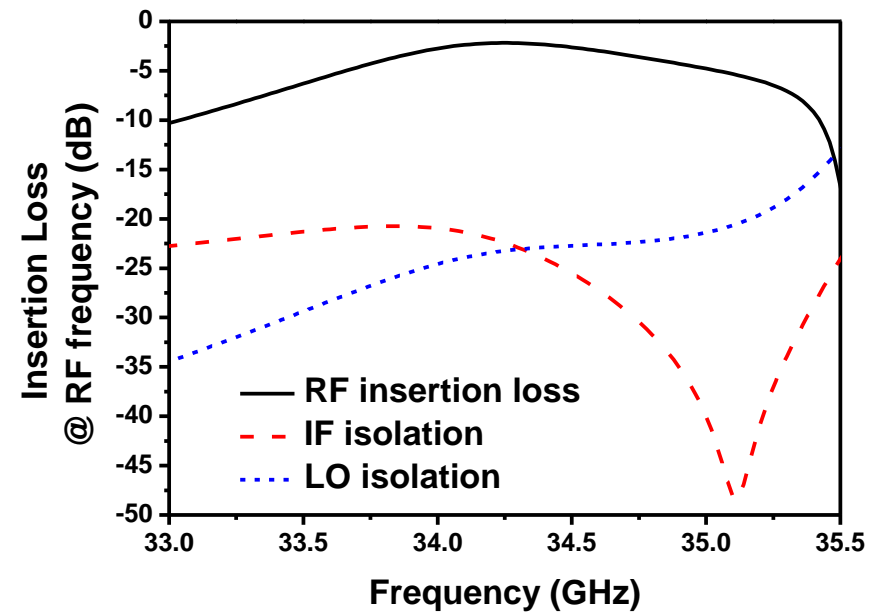

Fig. 3. Simulated insertion loss between the junction and the input/output ports at RF frequency ( $0.1 \mathrm{GHz}$ Frequency step)..

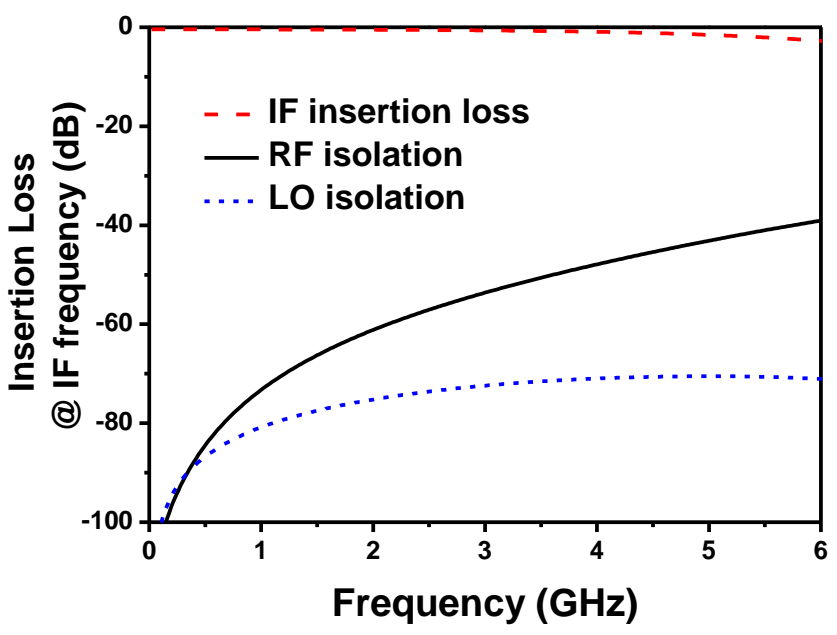

Fig. 4. Simulated insertion loss between the junction and the input/output ports at IF frequency $(0.1 \mathrm{GHz}$ Frequency step)..

mainly focused on RF-to-IF isolation, while the port impedance is still designed to be close to $50 \Omega$, as shown in Fig. 2. A lowpass filtering network is designed, consisting of a stub line $S_{1}$, a high impedance transmission line $L_{1}$, and the radius stub $\mathrm{RS}_{3}$ mentioned above. Additionally, a number of RF suppression method are applied for the $\mathrm{V}^{+}$and $\mathrm{I}_{\mathrm{B}}{ }^{+}$biasing line, including the shunted radius stub $\mathrm{RS}_{2}$ and the miniature RF blocking network outside the MMIC circuit. The biasing pad for $\mathrm{V}^{-}$and $\mathrm{I}_{\mathrm{B}}{ }^{-}$is designed to be connected to ground to provide physical earth for IF port. The biasing line itself will introduce low extra loss due to its relatively short electrical length for IF frequencies. Optimized insertion loss between the junction and IF port is around $0.5 \mathrm{~dB}$ below $3 \mathrm{GHz}$, and a high isolation is achieved between the junction and RF/LO ports, as shown in Fig. 4.

The designed mixer was subsequently fabricated on a singlesided $\mathrm{YBa}_{2} \mathrm{Cu}_{3} \mathrm{O}_{7-\mathrm{x}}(\mathrm{YBCO}$ ) film on $\mathrm{MgO}$ substrate using the CSIRO well established step-edge junction technology [14]. More detailed descriptions of the device fabrication procedures and mixer measurement set-up can be found in earlier papers [8-10]. 


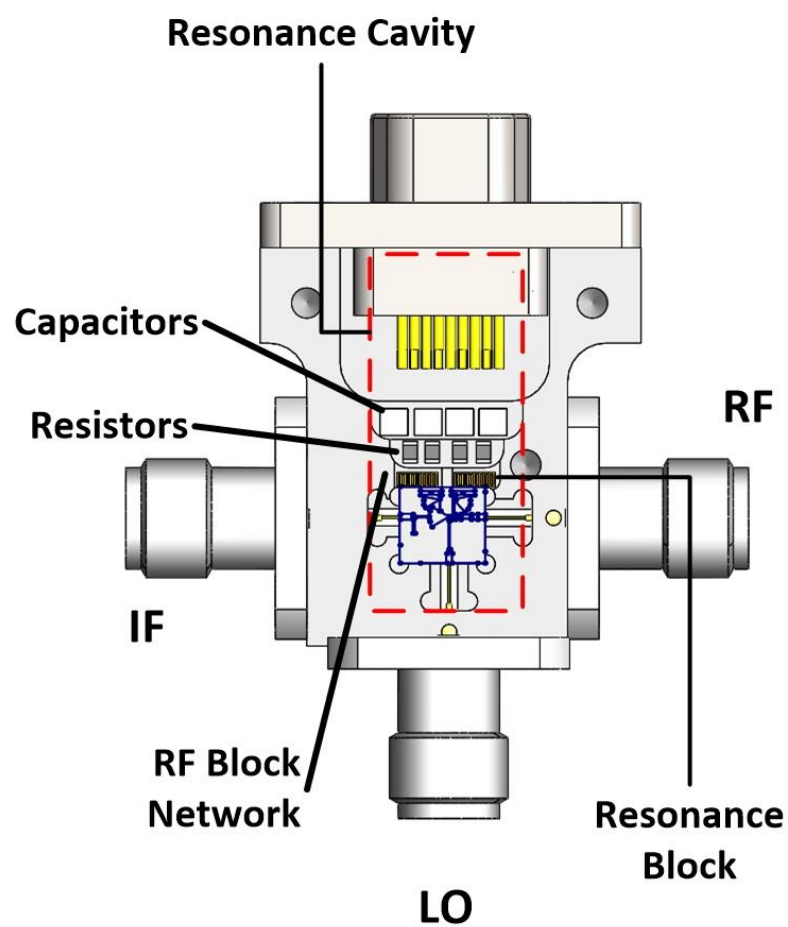

Fig. 5. Drawing of the packaged HTS Josephson mixer module.

\section{Module CONFIGURATION}

The fabricated HTS mixer chip was packaged into a customized metal housing, along with other lumped elements of DC biasing networks as shown in Fig. 5. The overall dimension of the module is below $25 \times 20 \times 15 \mathrm{~mm}^{3}$, which is very compact. For module designs at the frequency over $30 \mathrm{GHz}$, cavity resonance can affect the performance of the whole module and can no longer be neglected. Although the dimension of the Josephson mixer has been designed small enough to avoid cavity resonance around $34 \mathrm{GHz}$, small segments for the mixer, RF blocking networks, resistors and capacitors have formed a cavity with the dimension around $5 \times 10 \times 2 \mathrm{~mm}^{3}$, which would resonate at $33.5 \mathrm{GHz}$. Simulation in Fig. 6 (a) has shown that the cavity resonance at $33.5 \mathrm{GHz}$ does affect the HTS MMIC mixer area (Fig. 6 (a)). This may lead to severe deterioration in transmission loss and the shape of frequency response as reported in previous work [10]. To prevent the cavity resonance from affecting the performance of the mixer, a metal post was

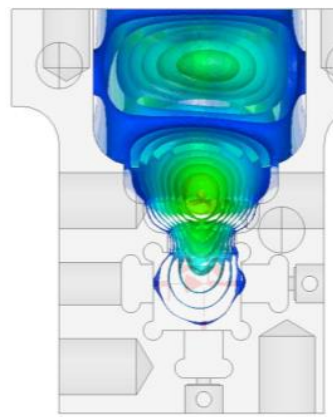

(a)

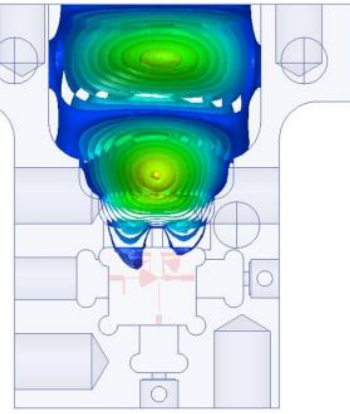

(b)
Fig. 6. Simulation of cavity resonance at $33.5 \mathrm{GHz}$ : (a) without and (b) with the resonance blocker. placed between the two pieces of RF blocking network circuits, as shown in Fig. 5. The post functions as an RF choke in the cavity, and has substantially altered the cavity resonance mode ( $\mathrm{TE}_{101}$ mode) in the approximate rectangular cavity in Fig. 6 (a). Due to the existence of the metal post, cavity resonance of the housing only occurs outside the HTS mixer chamber, as shown in Fig. 6 (b), which ensures that the mixer performance will not be affected by the housing.

\section{MEASUREMENT AND DisCUSSION}

The packaged module was cooled down to an operating temperature in $20-77 \mathrm{~K}$ with a commercial 2-stage pulse-tube cryocooler. A battery-driven DC current source was used to bias the Josephson device. The microwave RF signal generated by an Anritsu 68087C Synthesized CW Generator was applied to the RF port. An Agilent E4407B spectrum analyzer was connected to the IF port for output power measurement. The experimental results of the HTS receiver front-end at $40 \mathrm{~K}$ are presented here and compared with the simulated results.

Fig. 7 (a) is the measured I- V characteristic of the step-edge junction, with a partial $I_{\mathrm{c}}$ suppression when pumped by LO, and

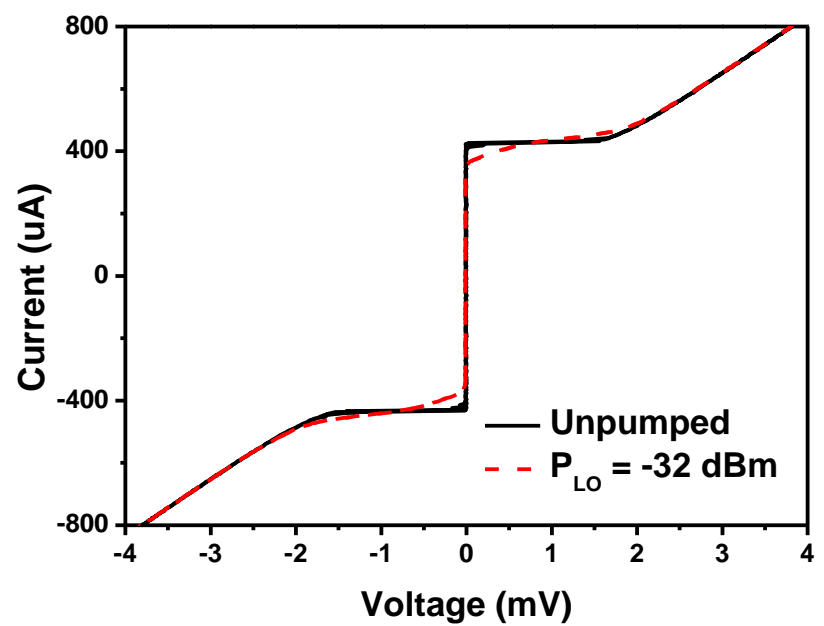

(a)

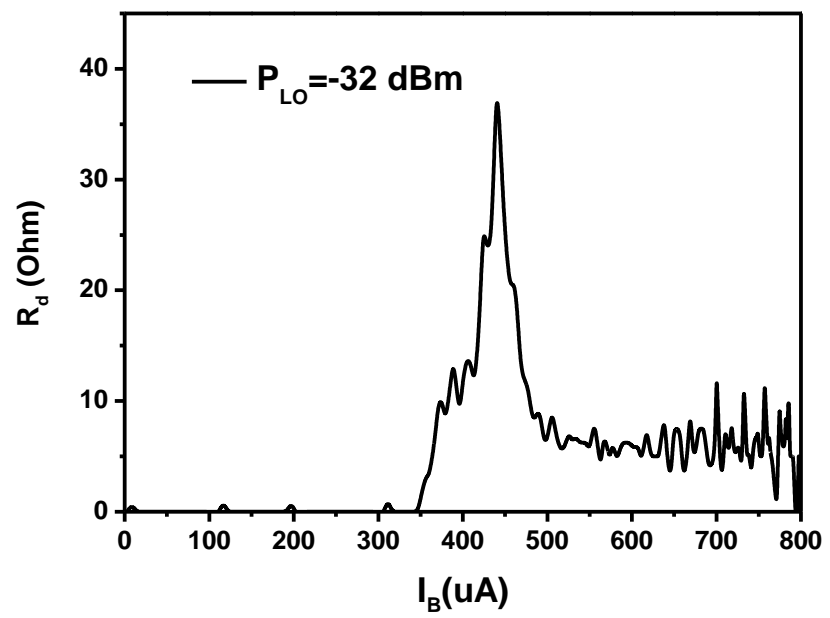

(b)

Fig. 7. (a) Measurement result of the junction DC I-V behavior, and (b) the derived dynamic resistance $\mathrm{R}_{\mathrm{d}}$ when pumped at $P_{\mathrm{LO}}=-32 \mathrm{dBm}$. 


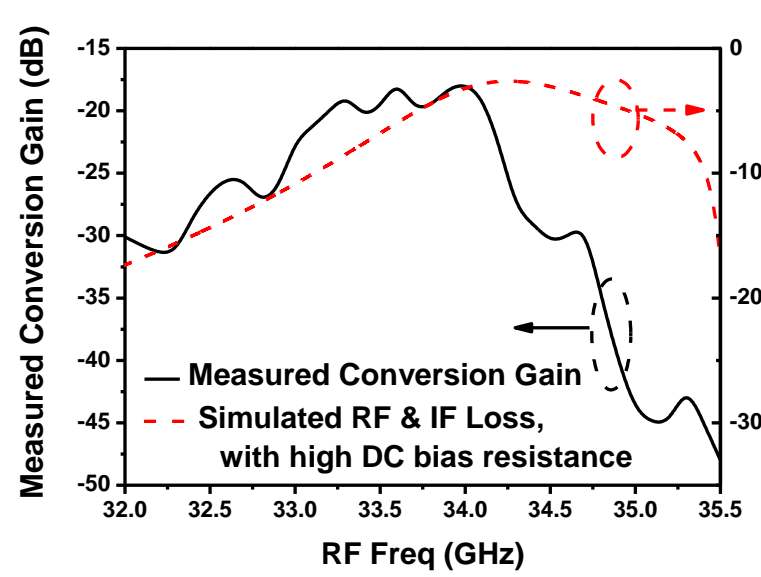

Fig. 8. Measured frequency response of the mixer conversion gain, and simulated frequency response of combined RF and IF loss $(0.1 \mathrm{GHz}$ Frequency step used)

(b) is its dynamic resistance $R_{\mathrm{d}}$ derived from the I-V behavior. The unsuppressed junction critical current $I_{\mathrm{c} 0}$ is around $450 \mu \mathrm{A}$, and is suppressed down to $360 \mu \mathrm{A}$ at $P_{\mathrm{LO}}=-32 \mathrm{dBm}$. The junction normal resistance $R_{\mathrm{n}}$, determined by the linear section of the I-V curve, is about $5 \Omega$, while the maximum $R_{\mathrm{d}}$ is around 40 $\Omega$. Both $\mathrm{R}_{\mathrm{n}}$ and $R_{\mathrm{d}}$ values are close to what has been anticipated and discussed in Section II. The $I_{\mathrm{c} 0} R_{\mathrm{n}}$ product obtained for this junction is around $2.2 \mathrm{mV}$, which is a high value compared with previous ones $[8,10]$.

Frequency response of the mixer IF output power was measured, and a calculated conversion gain of $-17 \mathrm{~dB}$ is obtained as shown in Fig. 8. For comparison, simulated frequency response of combined RF and IF transmission loss is also presented in the figure. Compared with the simulated transmission loss, which is around $4 \mathrm{~dB}$, the measured conversion gain is approximate $10 \mathrm{~dB}$ lower than that expected, with a sharper roll-off at the upper stopband around $34.5 \mathrm{GHz}$. The difference between the simulation and measurement results is believed to be due to the choice of the miniature RF blocking networks. From the supplier's datasheet [15], the RF blocking network circuit provides a cascade $10 \Omega$ resistor, and a shunted 30 pf capacitor, instead of components with high impedance. At IF frequencies, the shunted capacitor and the $10 \Omega$ resistors have induced a serious impedance mismatch between the IF port and the junction's dynamic resistance $R_{\mathrm{d}}$, thus resulting increased losses. This hypothesis has now been verified using the simulation approach of IF insertion loss described in Section II and the results are shown in Fig. 9 (a). After taking into consideration of the low value impedance of the RF blocking networks, the simulated insertion loss of IF link has increased by over $7 \mathrm{~dB}$ between the frequencies from 0 to $2 \mathrm{GHz}$, and the flatness of the frequency response has been severely deteriorated. Consequently, the overall transmission loss of the mixer has risen to $-14 \mathrm{~dB}$ with a sharp roll-off at the upper stopband as the simulated result shown in Fig. 9 (b). The measurement results agree well with the simulation ones having taken into consideration of the effect of the RF blocking network circuits, which proves that the proposed simulation approach of the HTS MMIC Josephson mixer is applicable. The performance of the mixer

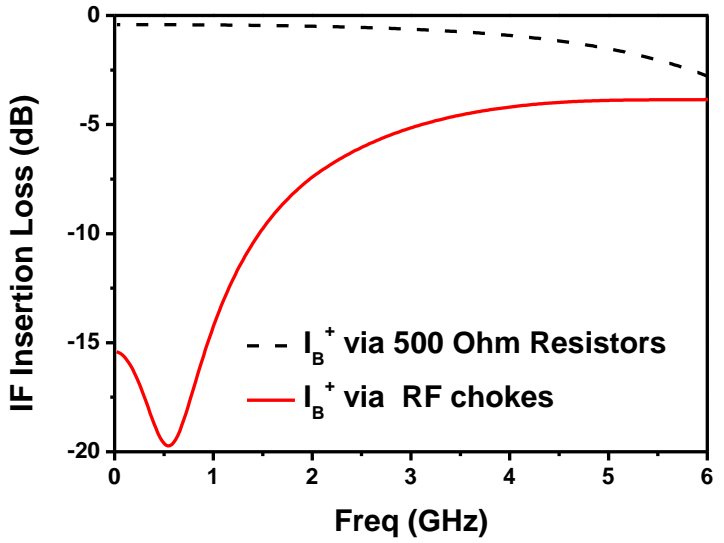

(a)

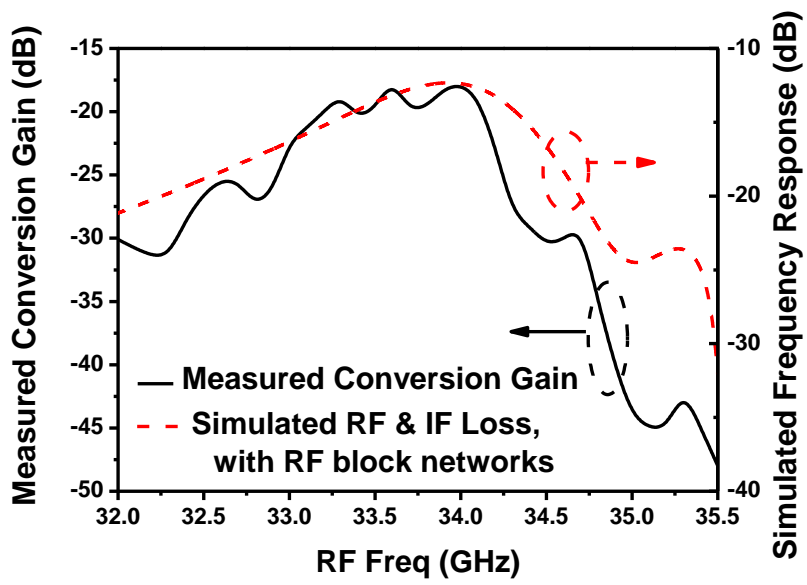

(b)

Fig. 9. (a) Simulated IF transmission losses comparing with a higher value resistance and the low impedance RF chokes for the assembled mixer, and (b) frequency response of the measured conversion gain and the simulated transmission response having taken into consideration of the low-impedance RF blocking networks ( $0.1 \mathrm{GHz}$ Frequency step used).

should improve by replacing the RF blocking network circuits with $1 \mathrm{k} \Omega$ resistors in future designs.

\section{CONCLUSION}

Full EM modeling and design of a Ka-band HTS MMIC Josephson mixer are presented in this paper. The junction area is modeled as an excitation port, with impedance of $R_{n}$ at RF frequency and IF frequency. In this way, transmission loss and impedance matching of the whole circuit can be simulated using the EM simulation software. Cavity resonance is eliminated in the module packaging design by inserting a metal post in the housing. Measured frequency response of conversion gain agrees well with the simulated transmission loss when taking into consideration of the effect of DC bias networks. The EM modeling and simulation method presented in this paper have been proven to be a good approach for HTS MMIC Josephson mixer design, which is a major improvement from the theoretical modeling developed previously [13]. 


\section{ACKNOWLEDGMENT}

We thank our colleagues, Mei Shen for packing and wire bonding, and Ms Jeina Lazar for HTS chip fabrication.

\section{REFERENCES}

[1] M. Klauda et al., "Superconductor and cryogenics for future communication systems," IEEE Trans. Microwave Theory Tech., vol. 48, pp. 1227-1239, Jul. 2000.

[2] R. R. Mansour, "Microwave superconductivity," IEEE Trans. Microw. Theory Tech., vol. 50, pp. 750-759, 2002.

[3] R. W. Simon, R. B. Hammond, S. J. Berkowitz, and B. A. Willemsen, "Superconducting microwave filter systems for cellular telephone base stations," Proc. IEEE, vol. 92, pp. 1585-1596, 2004.

[4] D. P. Butler, W. Y. Yang, J. Wang, A. Bhandari,and Z. Celik-Butler, "Conversion loss of a YBa2Cu307 grain boundary mixer at $20 \mathrm{GHz}$," Appl. Phys. Lett., vol. 61, p. 20, Jul. 1992.

[5] Y. Taur, J. Claassen, and P. Richards, "Josephson junctions as heterodyne detectors," IEEE Trans. Microw. Theory Techn., vol. 22, no. 12, pp. 1005-1009, Dec. 1974.

[6] K. Yamaguchi, A. Kawaji, K. Suzuki, Y. Enomoto, and S. Tanaka, "IF output characteristics of Josephson mixer," Electron. Comm. Jpn. 2, vol. 80, no. 3, pp. 69-79, 1997.

[7] X. Gao, T.Zhang, J. Du, A. R. Weily,Y. J. Guo, and C. Foley, "A Wideband Terahertz High-Tc Superconducting Josephson-Junction Mixer: Electromagnetic Design, Analysis and Characterization," Supercond. Sci. \& Technol., 30 (2017) 095011 (9pp).

[8] Ting Zhang, Jia Du, Yingjie Jay Guo, Xiaowei Sun, "A HighPerformance MMIC HTS Josephson Mixer", IEEE Trans Microwave and Wireless Components Lett., 23 (8) 427-429, 2013.

[9] J. Du, D. D. Bai, T. Zhang, Y. Jay Guo, Y. S. He and C. M. Pegrum, "Optimised Conversion Efficiency of a HTS MMIC Josephson Downconverter”, Supercond. Sci. \& Technol. 27 (2014) 105002 (8pp).

[10] T. Zhang, J. Du, J. Wang, D. D. Bai, Y. Jay Guo, and Y. S. He, “30 GHz HTS Receiver Front-end based on Monolithic Josephson Mixer", IEEE Trans Appl. Supercond., vol. 25, No. 3, Digital Object Identifier: 10.1109/TASC.2014.2379095, 2015.

[11] T. Zhang, X. Gao, W. Wang, J. Du, C. Pegrum, Y. J. Guo, “ A 36 GHz HTS MMIC Josephson Mixer - Simulation and Measurement," IEEE Transaction on Applied Superconductivity, vol. 27, no. 4, 1502405, Jan 2017.

[12] C. Pegrum, T. Zhang, J. Du, and Y. J. Guo, "Simulation of HTS Josephson Mixers," IEEE Transaction on Applied Superconductivity, vol. 26, no. 3, 1500905, Apr 2016.

[13] Ting Zhang, Colin Pegrum, Jia Du, Yingjie Jay Guo, "Simulation and measurement of a Ka-band HTS MMIC Josephson junction mixer ", Supercond. Sci. \& Technol.30 (1) 015008 (8pp).

[14] C. P. Foley, et al., "Fabrication and characterisation of YBCO single grain boundary step edge junctions," IEEE Trans. Appl. Supercond., vol. 9, pp. 4281-4284, 1999.

[15] Dielectric laboratories, RF blocking network, http://www.knowlescapacitors.com/dilabs/en/globalnavigation/products/ broadband-blocking-capacitors/rf-blocking-networks 